\title{
MAPPING OUT THE FULL SPIN RESONANCE STRUCTURE OF RHIC
}

\author{
V. Ranjbar,S.Y.Lee, Indiana University, Bloomington, IN \\ W. W. MacKay, M. Bai, E.Courant, BNL, Upton, NY
}

\section{Abstract}

We extended the ability of DEPOL [1] to calculate coupled spin resonances and used it to determine the location and strength of both intrinsic and coupled spin resonances in RHIC. In particular we are interested in the full resonance structure with solenoidal elements turned on and with quadrupole rolls[2]

\section{MODIFICATION OF DEPOL ALGORITHM}

Following the development of the DEPOL[1]algorithm which begins with the following general expression for resonance:

$$
\varepsilon=\frac{1}{2 \pi} \oint \frac{\zeta(s)}{\rho(s)} e^{i K \theta(s)} d s
$$

where $\zeta=-(1+G \gamma)\left(\rho z^{\prime \prime}+i z^{\prime}\right)+i \rho(1+G)(z / \rho)^{\prime}, \mathrm{K}$ is the resonance tune and $\theta$ is the orbital bending angle. This integral can be broken up into contributions from separate magnets:

$$
\begin{gathered}
\varepsilon=\sum_{\text {lattice }} \varepsilon_{m} \\
\varepsilon_{m}=-\frac{1}{2 \pi} \int_{s_{1}}^{s_{2}}\left\{(1+K)\left(z^{\prime \prime}+\frac{i z^{\prime}}{\rho}\right)\right. \\
\left.-i(1+G)\left(\frac{z}{\rho}\right)^{\prime}\right\} e^{i K \theta(s)} d s
\end{gathered}
$$

Applying partial integration to this integral we can obtain an intermediate form:

$$
\begin{array}{r}
\varepsilon_{m}=\frac{1}{2 \pi}\left[\frac{(1+K)\left(\xi_{1}+i\right)}{\rho} z_{1} e^{i K \theta_{1}}+\right. \\
\frac{(1+K)\left(\xi_{2}-i\right)}{\rho} z_{2} e^{i K \theta_{2}}-(1+K) \\
\left.\int_{s_{1}}^{s_{2}} z^{\prime \prime} e^{i K \theta} d s-\frac{K}{\rho^{2}}(K-G) \int_{s_{1}}^{s_{2}} z e^{i K \theta} d s\right]
\end{array}
$$

where $\xi$ are contributions due to edge focusing of the magnet. Applying partial integration again so that we obtain:

$$
\begin{gathered}
\varepsilon_{m}=\frac{1}{2 \pi}\left[\frac{(1+K)\left(\xi_{1}-i\right)}{\rho} z_{1} e^{i K \theta_{1}}+\right. \\
\frac{(1+K)\left(\xi_{2}-i\right)}{\rho} z_{2} e^{i K \theta_{2}}-(1+K)\left(\left(z_{2}^{\prime}-\frac{i K}{\rho} z_{2}\right) e^{i K \theta_{2}}\right. \\
\left.\left.-\left(z_{1}^{\prime}-\frac{i K}{\rho} z_{1}\right) e^{i K \theta_{1}}\right)+\left(\frac{K\left(K^{2}+G\right)}{\rho^{2}}\right) \int_{s_{1}}^{s_{2}} z e^{i K \theta} d s\right]
\end{gathered}
$$

In the uncoupled case this integral is evaluated using the homogeneous equation $z^{\prime \prime}=-k_{z} z$, substitution using this equation allows us to exactly evaluate Eq. 5. This is the primary algorithm used in DEPOL[1]. In the case of linear coupling however the homogeneous equation is no longer valid for all the elements. So we proceed by block diagonalizing the individual transfer matrices for the coupling element. Thus we hope to transform the $\mathrm{z}$ coordinate into a basis where a new homogeneous equation is true. The technology to accomplish this has been already developed by Edwards and Teng[3]. Thus given an element with off diagonal values in the $4 \times 4$ transfer matrix:

$$
M_{e}=\left(\begin{array}{cc}
A_{e} & B_{e} \\
C_{e} & D_{e}
\end{array}\right)
$$

Here A,B,C and D represent $2 \times 2$ submatricies which can be used to develop a transformation which will block diagonalize $M_{e}$ (the subscripts $e$ denote that only individual transfer matrices are being consider as opposed to the one turn transfer matrix):

$$
\begin{gathered}
R_{e} M_{e} R_{e}^{-1}=\left(\begin{array}{cc}
E_{e} & 0 \\
0 & F_{e}
\end{array}\right) \\
R_{e}=\frac{1}{\sqrt{1+\left|r_{e}\right|}}\left(\begin{array}{cc}
I & -\overline{r_{e}} \\
r_{e} & I
\end{array}\right) \\
r_{e}=-\left(\frac{1}{2}\left[\operatorname{Tr}\left(A_{e}\right)-\operatorname{Tr}\left(D_{e}\right)\right]+\right.
\end{gathered}
$$

$\left.\operatorname{sign}\left(\left|\mathrm{B}_{\mathrm{e}}+\overline{\mathrm{C}_{\mathrm{e}}}\right|\right) \sqrt{\left|\mathrm{B}_{\mathrm{e}}+\mathrm{C}_{\mathrm{e}}\right|+\frac{1}{4}\left(\operatorname{Tr}\left(\mathrm{A}_{\mathrm{e}}\right)-\operatorname{Tr}\left(\mathrm{D}_{\mathrm{e}}\right)\right)^{2}}\right)^{-1}$

Using $R_{e}$ we can express $x, p_{x}, z, p_{z}$ in a locally uncoupled basis $a, p_{a}, b, p_{b}$ (the over bar on $\mathrm{C}$ indicates a symplectic conjugate):

$$
\left(\begin{array}{c}
x \\
p_{x} \\
z \\
p_{z}
\end{array}\right)=R_{e}^{-1}\left(\begin{array}{c}
a \\
p_{a} \\
b \\
p_{b}
\end{array}\right)
$$

In this basis the homogeneous equations $a^{\prime \prime}=-k_{a} a$ and $b^{\prime \prime}=-k_{b} b$ will hold. We can determine $k_{a}$ and $k_{b}$ by considering that from accelerator theory:

$$
\left(\begin{array}{l}
a_{2} \\
a_{2}^{\prime}
\end{array}\right)=\left(\begin{array}{cc}
\cos \left(\varphi_{a}\right) & \frac{\sin \left(\varphi_{a}\right)}{\sqrt{k_{a}}} \\
-\sqrt{k_{a}} \sin \left(\varphi_{a}\right) & \cos \left(\varphi_{a}\right)
\end{array}\right)\left(\begin{array}{l}
a_{1} \\
a_{1}^{\prime}
\end{array}\right)
$$


Thus $k_{a}=-\frac{E_{e_{2,1}}}{E_{e_{1,2}}}$ and $k_{b}=-\frac{F_{e_{2,1}}}{F_{e_{1,2}}}$. So to solve the integral in equation 5 we can write $z=\left[r_{e_{1,1}} a+r_{e_{1,2}} p_{a}+\right.$ b] $\frac{1}{\sqrt{1+\left|r_{e}\right|}}$ to obtain:

$$
\begin{gathered}
\int_{s_{1}}^{s_{2}} z e^{i k \theta} d s=\frac{1}{\sqrt{1+\left|r_{e}\right|}}\left\{-r_{e_{1,1}} \int_{s_{1}}^{s_{2}} \frac{a^{\prime \prime}}{k_{a}} e^{i K \theta} d s\right. \\
-\int_{s_{1}}^{s_{2}} \frac{b^{\prime \prime}}{k_{b}} e^{i K \theta} d s+r_{e_{1,2}} \int_{s_{1}}^{s_{2}} a^{\prime} e^{i K \theta} d s
\end{gathered}
$$

Here we have substituted the homogeneous equation in for $a$ and $b$ and considered the normalized momentum $p_{a} \approx a^{\prime}$. Now using a integration technique similar to the original DEPOL[1] paper:

$$
\begin{gathered}
\int_{s_{1}}^{s_{2}} a^{\prime \prime} e^{i K \theta} d s= \\
\frac{\left(a_{2}^{\prime}-\frac{i K}{\rho} a_{2}\right) e^{i K \theta_{2}}-\left(a_{1}^{\prime}-\frac{i K}{\rho} a_{1}\right) e^{i K \theta_{1}}}{1-K^{2} / k_{a} \rho^{2}}
\end{gathered}
$$

we obtain a final closed expression:

$$
\begin{gathered}
\int_{s_{1}}^{s_{2}} z e^{i K \theta} d s=\frac{1}{\sqrt{1+\left|r_{e}\right|}}\left\{\left(\frac{i K}{\rho} r_{e_{1,2}}-r_{e_{1,1}}\right)\right. \\
\left(\frac{\left(a_{2}^{\prime}-\frac{i K}{\rho} a_{2}\right) e^{i K \theta_{2}}-\left(a_{1}^{\prime}-\frac{i K}{\rho} a_{1}\right) e^{i K \theta_{1}}}{k_{a}-K^{2} / \rho^{2}}\right)- \\
\left(\frac{\left(b_{2}^{\prime}-\frac{i K}{\rho} b_{2}\right) e^{i K \theta_{2}}-\left(b_{1}^{\prime}-\frac{i K}{\rho} b_{1}\right) e^{i K \theta_{1}}}{k_{b}-K^{2} / \rho^{2}}\right) \\
\left.+r_{e_{1,2}}\left(a_{2} e^{i K \theta_{2}}-a_{1} e^{i K \theta_{1}}\right)\right\}
\end{gathered}
$$

Thus our final expression for the resonance contribution from each magnet element is:

$$
\begin{aligned}
& \varepsilon_{m}=\frac{1}{2 \pi}\left[\frac{(1+K)\left(\xi_{1}-i\right)}{\rho} z_{1} e^{i K \theta_{1}}\right. \\
& +\frac{(1+K)\left(\xi_{2}-i\right)}{\rho} z_{2} e^{i K \theta_{2}} \\
& -(1+K)\left(\left(z_{2}^{\prime}-\frac{i K}{\rho} z_{2}\right) e^{i K \theta_{2}}-\left(z_{1}^{\prime}-\frac{i K}{\rho} z_{1}\right) e^{i K \theta_{1}}\right)+ \\
& \left(\frac{K\left(K^{2}+G\right)}{\rho^{2}}\right)\left(\frac { 1 } { \sqrt { 1 + | r _ { e } | } } \left\{\left(\frac{i K}{\rho} r_{e_{1,2}}-r_{e_{1,1}}\right)\right.\right. \\
& \left(\frac{\left(a_{2}^{\prime}-\frac{i K}{\rho} a_{2}\right) e^{i K \theta_{2}}-\left(a_{1}^{\prime}-\frac{i K}{\rho} a_{1}\right) e^{i K \theta_{1}}}{k_{a}-K^{2} / \rho^{2}}\right)- \\
& \left(\frac{\left(b_{2}^{\prime}-\frac{i K}{\rho} b_{2}\right) e^{i K \theta_{2}}-\left(b_{1}^{\prime}-\frac{i K}{\rho} b_{1}\right) e^{i K \theta_{1}}}{k_{b}-K^{2} / \rho^{2}}\right) \\
& \left.+r_{e_{1,2}}\left(a_{2} e^{i K \theta_{2}}-a_{1} e^{i K \theta_{1}}\right)\right]
\end{aligned}
$$

Of course for those elements which are already block diagonal we can neglect the local rotation to a diagonal basis and employ the original form:

$$
\int_{s_{1}}^{s_{2}} z e^{i K \theta} d s=\frac{\left(z_{2}^{\prime}-\frac{i K}{\rho} z_{2}\right) e^{i K \theta_{2}}-\left(z_{1}^{\prime}-\frac{i K}{\rho} z_{1}\right) e^{i K \theta_{1}}}{k_{z}-K^{2} / \rho^{2}}
$$

for substitution into equation 5 .

\section{IMPLEMENTATION IN CODE}

DEPOL derives its twiss parameters from MAD twiss output files and using them constructs the $z_{1,2}$ and $z_{1,2}^{\prime}$ values necessary to evaluate the resonance amplitude. However when MAD evaluates the twiss values under conditions of linear coupling it employs V1 and V2 coordinates which correspond to the bloc diagonal basis for the one turn transfer matrix. To correctly evaluate the resonance strength we must transform back to the original basis to obtain $x, p_{x}, z, p_{z}$. So in our code we read in values for the $\mathrm{R}$ matrix which is printed in the madout file, and use it to transform the $V 1, V 1^{\prime}, V 2, V 2^{\prime}$ back to the $x, p_{x}, z, p_{z}$ basis. From here we can then implement equation 15 for each element.

There is an additional complication in the implementation of this program which is an expression of the subtleties of equation 1 . In the original DEPOL code implicit use was made of the properties of the enhancement function which for $\mathrm{G} \gamma$ values on resonance yield the exact contribution to the resonance amplitude from one turn around all the elements in the accelerator lattice. However since we are dealing with a Fourier expansion in non-integer $\mathrm{K}$ values the actual integral in equation 1 must go from minus infinity to plus infinity. When evaluating resonances due to linear coupling an integral only once around the lattice will yield a superposition of enhancement functions due to both vertical and horizontal tunes. In this case we are forced to integrate equation 1 over many passes to obtain a good approximation for the resonance strength.

\section{RESULTS FROM ANALYSIS OF THE AGS}

We initially looked at the effect of increasing solenoidal strength values for the partial snake in the AGS. We plotted resonance strength as a function of coupling strength $C_{x}=\frac{\left|\Delta Q_{\min }\right|}{\left|\nu_{x}-\nu_{z}\right|+\sqrt{\left|\nu_{x}-\nu_{z}\right|^{2}+\left|\Delta Q_{\text {min }}\right|^{2}}}$. Compared to previous approximations [4] we expect the slope to be approximately equivalent to the original intrinsic resonance at that energy. As you can see from Figure 1 this is exactly what we found as we moved from $0 \%$ to $5 \%$ snake.

Next we investigated the behavior of the resonance strength as a function of skew quad strength. Using the measured $C_{x}$ values and the resonance strength with zero skew quad field strength we constructed an approximation of the coupling resonance strength using [4]. As you can see in Fig 2 this approximation compares favorably with our results. Differences begin to set in when considering coupling elements which significantly alter the tunes and when considering very weak resonances where the periodic structure of the coupling can contribute to resonance calculations. 


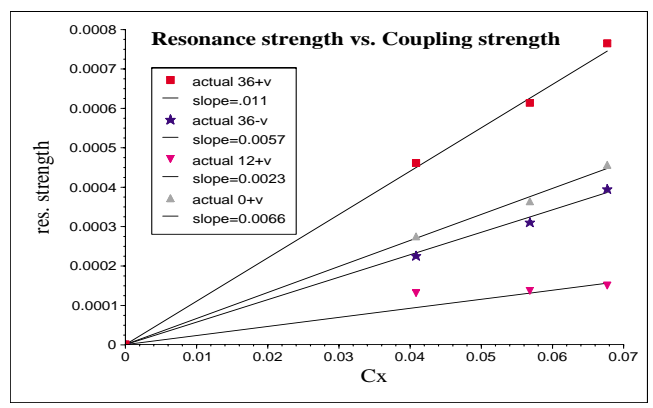

Figure 1: Spin coupling resonances vs. $\mathrm{C}_{x}$ for $36+\nu_{x}, 36-$ $\nu_{x}, 12+\nu_{x}$ and $0+\nu_{x}$ resonances. Where $\left(36+\nu_{z}\right)=0.01085$, $\left(36-\nu_{z}\right)=0.0057,\left(12+\nu_{z}\right)=0.0023$ and $\left(0+\nu_{z}\right)=0.0062$

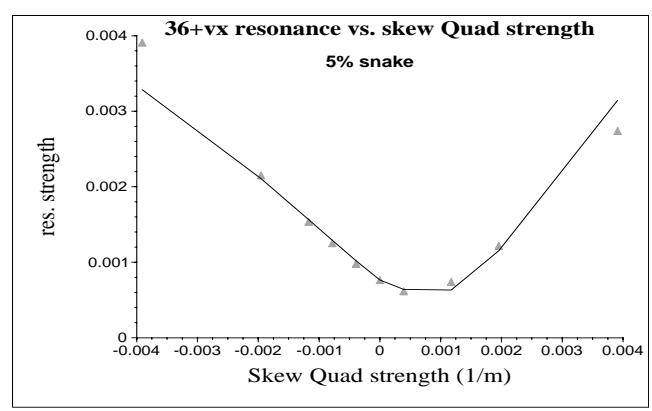

Figure 2: $36+\nu_{x}$ resonance strength vs. skew Quadrupole field strength with 5\% snake.The straight line is the approximation [4]

\section{PRELIMINARY RESULTS FROM RHIC}

In RHIC the coupling elements originate from three possible sources. Quadrupole rolls, solenoidal fields in the detectors and longitudinal fields in the snakes. Using preliminary values for the quadrupole rolls in the interaction region obtained from [2] we generated Fig. 3. In Fig. 4 we evaluated the Coupling spin resonances for the peak values for the solenoidal fields in the PHENIX and STAR detector.

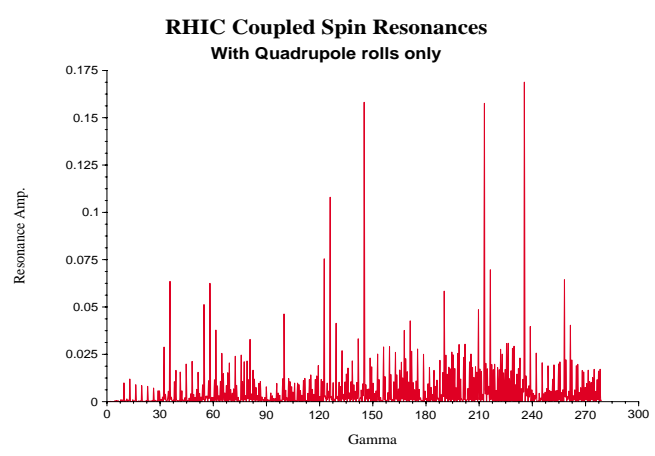

Figure 3: Modified DEPOL results on for Coupled Spin resonances on lattice with quadrupole rolls errors in interaction regions

In Fig 5 using the transfer matrices obtained from actual field maps of the helical snakes [5], you can see the contri-

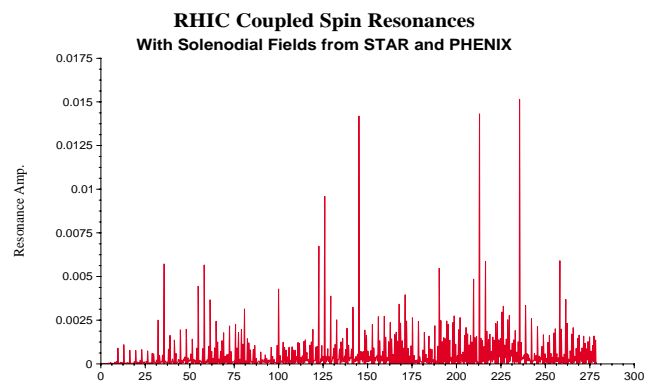

Figure 4: Modified DEPOL results on for Coupled Spin resonances on lattice with solenoidal fields from PHENIX and STAR turned on

bution to the Coupling spin resonance from a single snake element. While clearly the function of the snake is to eliminate resonances thus Fig 5 doesn't give the whole picture it does provide a useful tool to better understand the strength and location of potential snake resonances.

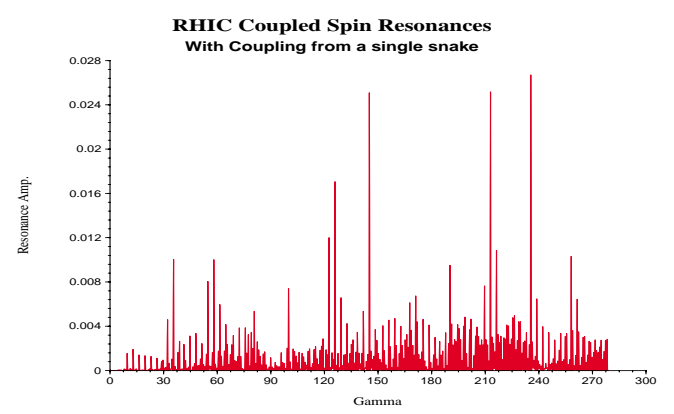

Figure 5: Modified DEPOL results on for Coupled Spin resonances on lattice with coupling from a single helical snake.

\section{REFERENCES}

[1] E.D. Courant and R.D. Ruth, The Acceleration of Polarized Protons in Circular Accelerators, BNL 512701980.

[2] Cardona Javier, personal communication

[3] L.C. Teng, Concerning n-Dimensional Coupled Motion. FN 229, FNAL, 1971.

[4] S.Y.Lee, "Spin Dynmanics and Snakes in Synchrotrons", World Scientific 1997,pg. 34.

[5] V.Ranjbar,W.Mackay,A,Luccio,N.Tsoupas, Fitting Helical Snake and Rotator field strength measurements in RHIC, to be published PAC 2001 paper. 